Archives de sciences sociales des religions

$177 \mid 2017$

Mondes juifs en mouvement

\title{
Juifs d'Istanbul : du parcours minoritaire aux porosités exclusives
}

Istanbul's Jews: from a minority trajectory to exclusive porosities

Judíos de Estambul: del recorrido minoritario a las porosidades exclusivas

\section{Yoann Morvan}

\section{(2) OpenEdition}

\section{Journals}

Édition électronique

URL : http://journals.openedition.org/assr/29287

DOI : $10.4000 /$ assr.29287

ISSN : $1777-5825$

Éditeur

Éditions de l'EHESS

Édition imprimée

Date de publication : 1 mars 2017

Pagination : 115-132

ISSN : 0335-5985

Référence électronique

Yoann Morvan, « Juifs d'Istanbul : du parcours minoritaire aux porosités exclusives », Archives de sciences sociales des religions [En ligne], 177 | 2017, mis en ligne le 01 mars 2019, consulté le 06 janvier 2021. URL : http://journals.openedition.org/assr/29287; DOI : https://doi.org/10.4000/assr. 29287 


\section{Juifs d'Istanbul : du parcours minoritaire aux porosités exclusives}

Minorité historique de l'Empire ottoman et de sa capitale, les Juifs d'Istanbul ont connu trois strates principales de profondes transformations autant territoriales qu'identitaires entre la fin du XIX ${ }^{e}$ siècle et nos jours. Ainsi, la minorité juive stambouliote a vécu, durant les siècles de l'Empire, dans le «système des millet ", ce qui lui a conféré des droits et des devoirs spécifiques: un certain nombre de contraintes, seulement négociables à la marge. Puis, avec l'avènement de la République turque à la suite de la Première Guerre mondiale, les Juifs d'Istanbul ont dû s'adapter à un nouveau contexte notamment statutaire, qui en apparence assigne moins, mais se trouve être moins protecteur. Enfin, l'évolution de la donne politique et culturelle en Turquie et le progressif abandon du modèle kémaliste vont contribuer à modifier encore les positions possibles des Juifs au sein de la société urbaine stambouliote.

On voudrait ici analyser la manière dont chacune de ces strates (idéaltypiques) a pu générer des types de frontière ad hoc ainsi que des façons de les franchir. Notre attention particulière s'attache à appréhender ces frontières dans leurs territorialités, aux interactions réciproques entre sociétés et formes urbaines. En effet, il n'est pas indifférent aux dynamiques frontalières que tel groupe réside majoritairement dans un quartier "traditionnel ", dans un parcellaire d'avenues/rues/îlots/immeubles ou encore dans des gated communities, etc. À chacun de ces espaces résidentiels correspondent des logiques sociales spécifiques avec leurs fermetures et porosités.

L'intérêt du cas étudié ici provient du fait que ces trois strates ne sont pas strictement chronologiques. Elles continuent à jouer leur partition propre, superposant les uns aux autres des registres de frontières, qui suscitent non seulement des apories et des contradictions mais aussi des créativités dans la capacité des Juifs d'Istanbul à redéfinir leur(s) groupe(s), en pluralisation croissante. 


\section{Ghettos minoritaires?}

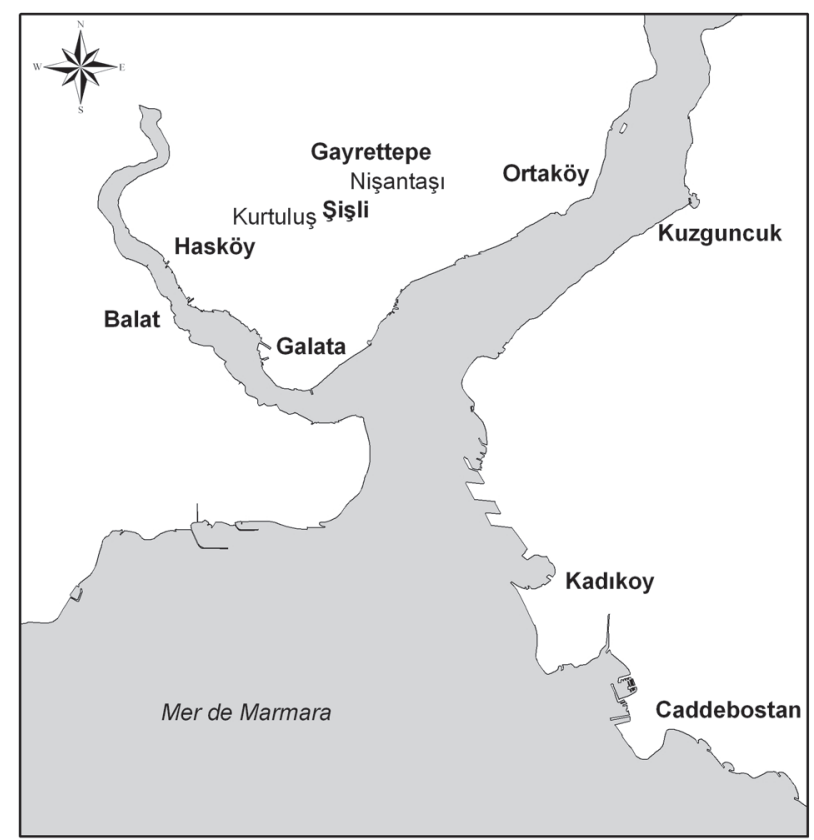

Photo 1. Mahalle et köy à forte présence juive ( $\mathrm{XIX}^{\mathrm{e}}-\mathrm{XX}^{\mathrm{e}}$ siècle), carte de l'auteur

\section{Territorialités de la millet juive à Istanbul}

Sans remonter à la principale vague d'installation dans l'Empire ottoman des Juifs séfarades aux lendemains de l'Inquisition (1492), nous nous bornerons ici à décrire la géographie de la présence juive à Istanbul au tournant des $\mathrm{XIX}^{\mathrm{e}}-\mathrm{XX}^{\mathrm{e}}$ siècles, cela afin de tenter d'éclairer quels étaient alors les gradients de fermeture/porosité de la minorité vis-à-vis de ses environnements tant résidentiel que professionnel.

Les Juifs d'Istanbul se sont principalement agglomérés dans deux quartiers historiques de la métropole ottomane jouxtant la Corne d'Or (Balat, Hasköy), dans un certain nombre de villages le long du Bosphore (Ortaköy, Yeniköy, Kuzguncuk) (Karmi, 1992), ainsi que dans de nouveaux points de fixation urbaine (Galata, Kadiköy) qui annoncent déjà la strate ultérieure. Ce semis territorial, en évolution du fait de la modernisation de l'Empire et de sa capitale à cette période, se voit matérialisé par les synagogues (Güleryüz, 2008). En effet, les Juifs d'Istanbul sont à cette époque encore largement pratiquants. Cela implique que leur habitat ne soit pas trop éloigné de ces lieux de culte, en particulier afin de pouvoir se rendre à pied à la synagogue lors du shabbat. 
Le faubourg de Balat, auquel Marie-Christine Varol a consacré une monographie (1989), est un exemple archétypique de cette géographie religieuse avec ses nombreuses synagogues dénommées en fonction des origines (surtout balkaniques) de leurs fidèles.

Si la minorité [millet] juive d'Istanbul a pendant longtemps résidé dans des quartiers [mahalle] spécifiques, ces derniers ne paraissent pas avoir constitué des ghettos, ne serait-ce que parce que ces espaces résidentiels n'étaient en général pas ceints de murs et jamais exclusivement habités par l'une des millet (Seni, 2006). La meilleure étude sur la notion et la pratique du quartier a été réalisée par l'historienne Noémi Lévy-Aksu, dans Ordre et désordres dans l'Istanbul ottomane (1879-1909) (2013). Rappelant les difficultés à définir le quartier et le regain d'intérêt que celui-ci connaît au sein de l'historiographie, elle montre bien à quel point c'est un échelon essentiel de la gouvernance urbaine et comment la vie interminoritaire n'y était pas toujours pacifique. S'appuyant sur les différences religieuses réelles et fantasmées, les querelles trouvent la plupart du temps leurs racines dans des différends commerciaux ${ }^{1}$, en particulier entre Grecs [Rums] et Arméniens d'un côté et Juifs de l'autre ${ }^{2}$. Ces «ordres" et "désordres" constituent des frontières démarquant les territorialités locales des uns et des autres, et révèlent également les rapports différenciés à l'autorité politique surplombant ces millet.

\section{Devenirs (néo-)ottomans et enclavement subi aujourd'hui}

À l'instar du quartier [mahalle] et pour des raisons convergentes, le système des millet a été appréhendé avec une curiosité renouvelée au cours des dernières années (Barkey, Gravilis, 2016). Si l'ouvrage de synthèse publié par Benjamin Braude et Bernard Lewis (1982) adoptait déjà un regard plutôt positif à leur égard, la tendance s'est renforcée depuis (Levy, 2002), jusqu'à devenir un lieu commun de nos jours. Cet éloge rétrospectif des millet s'inscrit dans un courant qui vise plus généralement à promouvoir la période ottomane, en particulier ses prétendues vertus de «tolérance» en matières politique, religieuse et culturelle. La rhétorique de ce courant, porté par le parti issu de la mouvance de l'islam politique et actuellement au pouvoir en Turquie (AKP), a été partiellement assimilée et reprise par la communication de la communauté juive d'Istanbul, au prix parfois d'exagérations manifestes telle celle de cette publication intitulée: The Turkish Jews, 700 Years of Togetherness (Güleryüz, 2009). Le musée célébrant le 500e anniversaire de l'arrivée massive (1492) des Séfarades dans l'Empire ottoman incarne lui aussi ce tropisme: «"Amitié judéo-turque”, "paix”, "liberté religieuse”, “coexistence”, “respect

1. Ces relations commerciales intercommunautaires sont d'ailleurs assez mal connues dans le détail. Il s'agirait de tester les stimulantes hypothèses formulées par Francesca Trivilatto (2009) à propos du cas livournais, en particulier la notion de «communitarian cosmopolitanism ".

2. Une partie de ces débats a été faussée par des historiographies partisanes, notamment anti arméniennes. 
mutuel", "égalité", "humanisme" et "civilisation" abondent dans les textes des panneaux d'affichage ", pointe l'anthropologue Marcy Brink-Danan; selon cette dernière, "plutôt que d'exiger des droits [...], leurs représentants officiels demandent [...] la reconnaissance, le consentement et les faveurs » (Brink-Danan, 2010a). Les Juifs d'Istanbul reproduisent ainsi leur ethos de millet, plus d'un siècle après...

L'enracinement de cette attitude, qui se caractérise notamment par le "profil bas", est ancien au sein de la minorité juive d'Istanbul. La fin de l'Empire ottoman constitue l'une des périodes clé dans l'adoption de cette posture. En effet, le contexte des relations entre les millet et les autorités de l'Empire changent à cette époque (fin XIX ${ }^{\mathrm{e}}$ ). Tandis que ce dernier est de plus en plus suspicieux à l'égard des Grecs et des Arméniens, les Juifs font tout pour faire figure de "minorité modèle (Cohen, 2014). Cette volonté d'apparaître toujours plus loyaux est une stratégie qui présente d'évidentes limites puisque les Juifs n'en sont pas moins considérés, quoi qu'il arrive, comme des «invités", voire, au gré des circonstances, comme des hôtes ingrats ${ }^{3}$... C'est du reste ce que n'ont pas manqué de rappeler certaines autorités turques lors de pics du conflit israélo-palestinien, synonymes de détériorations des relations turco-israéliennes. Impliquant bien des silences, le prix à payer de cette loyauté est assez exorbitant!

Ainsi, les Juifs d'Istanbul vivent jusqu'à aujourd'hui dans un enclavement partiellement subi et intériorisé. En effet, pour assurer sa sécurité, la minorité se voit contrainte de surprotéger ses lieux communautaires. Leurs entrées sont placées sous double surveillance: policière et privée (Morvan, 2011b). Elles ressemblent à des sas d'embarquement pour des vols internationaux (Brink, Danan, 2005). Un exemple particulièrement significatif de cette spatialisation de l'enclavement est fourni par la synagogue d'Ortaköy, ancien village au bord du Bosphore. Ainsi, la forme urbaine du lieu de culte est révélatrice du caractère centrifuge de la vie communautaire: il s'agit d'un îlot introverti au cœur du parcellaire du mahalle. Cette forme urbaine fermée, héritière de l'Empire ottoman, a été mise sous cloche de verre renforcée à la suite du double attentat perpétré en 2003 (dans les synagogues de Sisli et de Neve Şalom). Malgré les petites ouvertures laissées pour que les arbres de cette synagogue, dénommée Etz Ha 'Haïm [arbre de vie], prolongent leur existence, plus de la moitié ont fini par succomber... Lors du festin organisé pour la fête de Pourim en 2016, les opulentes grillades de diverses viandes ont, quant à elles, totalement enfumé l'espace de l'ancien patio autrefois à ciel ouvert... Cette atmosphère d'auto-étouffement marque également la vie sociale. La nostalgie de l'insularité enchantée qui caractérise le sentiment de nombreux Juifs d'Istanbul à propos des estivages sur les îles au Prince s'est transformée par ailleurs en hantise pour les plus jeunes générations, lasses du (trop) fort contrôle social interne qui y règne.

3. La communauté reproduisant cette attitude, j'ai moi-même été plusieurs fois soupçonné durant mon enquête de terrain. 
Le mixte d'enclavement et de néo-ottomanisme provoque deux effets complémentaires et, à première vue, paradoxalement convergents sur la minorité juive d'Istanbul: un effacement progressif, ne serait-ce qu'en raison du déclin inexorable de sa démographie ${ }^{4}$, doublé d'une visibilité sur commande. Cette mise en visibilité s'est notamment manifestée lors de l'année 2010, durant laquelle Istanbul a été "Capitale européenne de la culture». Ainsi, plusieurs événements se sont déroulés dans les lieux de la minorité, telle l'installation minimaliste d'art contemporain «Molecular» dans l'ancienne synagogue de la Mayor à Hasköy ${ }^{5}$, détruite en 1923 par un incendie et occupée depuis par des ateliers d'artisans (Morvan, 2011b). Un concert dénommé «Intersection », entendant ainsi magnifier la vocation de creuset culturel de la métropole ${ }^{6}$, s'est déroulé quant à lui dans la synagogue italienne, toujours en activité le samedi matin et lors des fêtes juives (Morvan, 2011a). Le cas des activités des synagogues de Kuzguncuk est également significatif de ce double mouvement d'effacement et de mise en visibilité à des fins politico-symboliques. Tandis que le quartier n'abrite plus que quelques très rares Juifs, les deux synagogues y connaissent une intense activité. En plus du calendrier juif qui voit bon nombre d'originaires du quartier revenir y célébrer le culte dans une joviale atmosphère aux accents nostalgiques, les synagogues accueillent de nombreux événements musulmans, en particulier des ruptures de jeûne du ramadan (iftar). Participant d'un prétendu dialogue judéo-musulman, ces événements servent de faire valoir aux autorités municipales stambouliotes et assurent de leur part une tolérance en apparence bienveillante ${ }^{7}$ à l'égard de la minorité juive locale. Le quartier, dorénavant gentrifié et vidé de ses minoritaires, se situe dans l'arrondissement d'Üsküdar, haut lieu de l'Islam à Istanbul dans les imaginaires musulmans et l'un des fiefs de l'AKP. La minorité juive d'Istanbul se voit de facto contrainte de jouer ce jeu de dupes au sein de

4. La diminution du nombre des Juifs en Turquie s'est réalisée par étapes: 82000 en 1927, puis 77000 en 1948, ils ne sont plus que 46000 en 1951, trois ans après la création de l'État d'Israël, et passent à 38000 en 1965 (Bali, 2009). Ce déclin doit être mis en perspective avec les évolutions démographiques, selon des chronologies un peu différentes, des anciennes minorités grecque (100 000 en 1927, 47000 en 1965 contre environ 4000 de nos jours), ou arménienne (30 000 rejoints par un nombre comparable de migrants venus d'Arménie récemment), et surtout avec l'accroissement considérable de la population turque à Istanbul comme dans l'ensemble du pays (24 millions en 1955 et 77 millions aujourd'hui).

5. Elle se situe à proximité d'une autre synagogue qui, après avoir servi de magasin de goudron puis de fonderie, a été cédée par la municipalité de Beyoğlu à un investisseur privé, qui l'a rénovée et transformée en café touristique. Le destin de ces édifices cultuels témoigne de la difficulté de la conservation du patrimoine dans une vocation autre que touristique. "Bâti orphelin, malmené ", faisant l'objet de «spoliations spontanées », notamment pour faire des parkings, comme la synagogue Kastorya à Balat de l'autre côté de la Corne d'Or, les biens non-musulmans sont soumis à rude épreuve une fois les territorialités désinvesties, les présences «en creux» (Pérouse, 2005).

6. Le maintien d'une présence juive à Istanbul concourt à son européanisation (Brink-Danan, 2010b).

7. L'efficacité de cette mise en scène politico-religieuse est telle que cela a même conduit une chercheuse américaine à quasiment prendre ce discours pour argent comptant (Mills, 2010). 
cette nouvelle économie symbolique "néo-ottomanisante". Le summum de théâtralisation des rapports politico-religieux trouve son incarnation dans la figure du grand rabbin ('Hakham Bashi) de Turquie aujourd'hui. Alors que celle-ci avait perdu de sa prestance durant la période kémaliste et son idéologie de laïcisation à marche forcée, cette figure paraît reprendre des couleurs et du lustre à l'ère néo-ottomane. Ce retour, un brin pastiche, aux millet s'affiche dans des vidéos diffusées dans le métro: elles représentent le 'Hakham Bashi en costume d'apparat ouvrant une porte.

\section{Le miroir aux alouettes de la turquification}

Revenons à la strate ultérieure à celle de la fin de l'Empire ottoman : le passage à la République turque et ses conséquences sur les Juifs d'Istanbul et leurs frontières identitaires et urbaines.

\section{Les avatars du kémalisme}

L'avènement de la République a constitué un bouleversement radical, non seulement pour ce qui est des rapports entre l'État et les minorités mais aussi sur la place d'Istanbul dans les représentations et la nouvelle configuration du pouvoir. En effet, considérée comme l'une des causes de la décadence de l'Empire, en particulier en tant que lieu de l'intrusion étrangère, notamment via les minorités, la métropole du Bosphore se voit destituée de son statut de capitale au profit d'Ankara (Pérouse, 2010). À la suite des négociations du traité de Lausanne, l'adoption du Code civil le 17 février 1926 met un terme aux millet, exerçant en amont des pressions sur les minorités pour qu'elles acceptent d'abandonner de leur plein gré ce statut. L'objectif est de les turquiser. Cette politique comporte deux volets principaux. L'un est culturel, il vise à l'application du triptyque «langue unique, culture unique, idéal unique », dont l'impact sur les Juifs d'Istanbul est plus fort que sur les autres minorités car, à la différence de l'arménien et du grec, leur langue, le judéo-espagnol, n’est pas reconnue du tout (Bali, 2001). L'autre est économique: «la turquisation a consisté à limiter le nombre d'employés des communautés minoritaires dans les entreprises à capital étranger. Jusqu'en 1923, 90 \% des cadres de ces entreprises étaient non-musulmans. En 1923, on a imposé à toutes ces entreprises d'employer $50 \%$ de personnel musulman et annoncé que celles qui ne rempliraient pas cette condition seraient fermées [...] La distinction entre musulmans et non-musulmans s'est surtout manifestée dans le secteur public qui n'embauchait pas de non-musulmans»(Bali, 2001). Cette politique discriminatoire atteint son paroxysme avec l'impôt sur la fortune [varlik vergisi] qui entre en vigueur en novembre 1942. Celle-ci finit de compléter le dispositif de turquification économique et s'apparente à une forme de spoliation des minorités, touchant plus particulièrement la minorité juive (Bali, 2009). Si la condition minoritaire a cantonné le destin économique des Juifs d'Istanbul au sein de l'espace social de la métropole durant la période ottomane, le passage 
à la République, sous couvert d'égalitarisme formel, vient quasiment aggraver le niveau de ségrégation à leur encontre. L'heure est à la constitution d'un bloc national homogène... $\operatorname{turc}^{8}$ !

Malgré la perte de son statut de capitale, le coup d'arrêt que cela a représenté et la décrue démographico-économique qui s'en est suivie, Istanbul continue sa lente marche vers la modernisation (Pérouse, 2010). Déjà engagée dans ce processus durant la fin de la période ottomane, l'élite juive stambouliote l'accompagne, d'un point de vue identitaire autant qu'urbain. Elle tente le jeu de la turquification et délaisse ses quartiers traditionnels au profit de nouvelles polarités urbaines. Dès la fin du XIX ${ }^{\mathrm{e}}$ siècle, notamment par le biais de l'enseignement en français dispensé par l'Alliance israélite universelle, les couches supérieures avaient d'elles-mêmes renoncé au judéo-espagnol, synonyme pour elles d'arriération culturelle et entravant la mobilité sociale. Elles poursuivent alors les ouvertures linguistiques qu'elles avaient entamées grâce à leurs voisinages, à leurs apprentissages divers et/ou à leurs activités professionnelles locales et internationales : turc, grec, arménien, hébreu, français, anglais, etc. Pas encore victimes de la réduction linguistique qu'a fini par constituer l'assimilation par la turquification, l'âge d'or de ce polyglottisme est le demi-siècle allant de 1910-1920 à 1950-1960: des routes commerciales anatoliennes au lycée francophone Notre Dame de Sion ou des intérieurs londoniens aux stambouliotes rues cosmopolites de Péra, le roman de Mario Levi, Istanbul était un conte (2011), en décrit avec justesse les territorialités (Morvan, 2011b).

Les parcours résidentiels des Juifs d'Istanbul à cette époque corroborent leur désir d'intégration aux évolutions urbaines de la métropole vers la modernité. Riva Kastoryano (1992b) emploie un joli jeu de mot turc pour décrire leur trajectoire comme un "mouvement vers le haut", signifiant ainsi la double ascension, sociale et spatiale. En effet, côté européen de la métropole du Bosphore, ces trajectoires suivent l'axe nord de développement de l'agglomération (Köksal, 2010): partant de Galata pendant le premier quart du $\mathrm{Xx}^{\mathrm{e}}$ siècle, elles se prolongent dans le temps et l'espace quasi immédiatement vers Taksim puis, en fonction des classes sociales, Şişli, Kurtuluş et Nişantaş, avant et après la Seconde Guerre Mondiale, puis enfin Gayrettepe. Au cours de ces mouvements migratoires à l'intérieur d'Istanbul, les points de passage et de redistribution des Juifs d'Istanbul sont au premier chef Galata ${ }^{9}$, puis Şişli ainsi que Kadiköy (coté asiatique). Bien que ces populations y soient assez regroupées, ce sont des étapes de désenclavement choisi. Reprenant le modèle de l'écologie urbaine conçu par l'École de Chicago, Kastoryano (1992b) décrit le mécanisme de push and pull interne et externe à la communauté juive: chaque nouvelle arrivée de populations pauvres, Juifs ou Turcs d'Anatolie,

8. Ce caractère turc comprend un versant linguistique mais aussi implicitement religieux: musulman sunnite.

9. En témoignent les multiples synagogues, dont Neve Şalom, ainsi que la présence du grand rabbinat. 
fait fuir les populations installées préalablement et prospères vers des secteurs urbains plus valorisés. Galata est un exemple de «stade de transition », selon le modèle de Louis Wirth. Galata a constitué le premier accès aux immeubles à appartements, synonymes de modernité et instaurateurs de fines distinctions sociales, d'une rue ou d'un bâtiment à l'autre. Cette forme urbaine sera la norme jusqu'à Gayrettepe côté européen et Bostanc1 côté asiatique. D’une certaine manière et outre son caractère universel, elle matérialise dans l'espace urbain l'homogénéisation kémaliste décrite plus haut.

\section{Les Juifs d'Istanbul ne seront jamais des « Turcs blancs »!}

Ces trajectoires résidentielles témoignent également d'un alignement de l'ancienne minorité sur ceux que l'on désigne comme les «Turcs blancs » [Beyaz Türkler]. Cette appellation qui apparaît au cours des années 1990, période à laquelle les populations issues l'exode rural (dénommées par contraste «Turcs noirs " [Zenci Türkler] par les premiers) commencent à figurer aux premiers plans des scènes socio-économique et politique à Istanbul. Les "Turcs blancs ", musulmans se voulant/disant "laïcs » et "européanisés », représentent les fers de lance du maintien de l'idéal kémaliste. L'identification territoriale et morale avec cette élite turque est particulièrement frappante à Caddebostan le long de l'avenue de Bagdad coté asiatique. Longtemps destination d'estivage avec ses maisons en bois avec jardin, ce secteur urbain est devenu assez central dans l'agglomération stambouliote à la faveur de l'ouverture des deux ponts sur le Bosphore (1973, 1988) (Montabone, Morvan, 2011). Cet axe d'urbanisation constitue en quelque sorte le pendant, du côté asiatique, de l'axe nord du côté européen. Il s'origine à Kadiköy, «l'autre ville de l'autre côté » (Akbulut, 2010), et se prolonge désormais bien au-delà de l'avenue de Bagdad, jusque vers Pendik. Si les Juifs d'Istanbul se sont égrainés tout le long de ce vecteur d'urbanisation, ce sont les alentours de Caddebostan qui concentrent leur écrasante majorité avec environ un millier de foyers (Morvan, 2011b). En général d'origine un peu plus modeste que la plupart de ceux du côté européen, exceptés certains secteurs comme Kurtuluş, les Juifs de Caddebostan se caractérisent, globalement et idéal-typiquement, par une plus grande homogénéité religieuse (traditionalistes assez pratiquants et affichage laïc) et socioculturelle (classes moyenne à supérieure) que leurs homologues du reste d'Istanbul; homogénéité qui elle-même définit ce secteur de la métropole, tant par ses formes urbaines (immeubles de six à dix étages et trame urbaine hyper régulière) que par les lifestyles de leurs habitants, notamment très friands de consommation: l'avenue de Bagdad est devenu une sorte de shopping mall à ciel ouvert. Il s'agit d'un nouvel épicentre de l'arrondissement de Kadiköy, le principal et à présent l'un des rares bastions à Istanbul du CHP, le parti kémaliste. Caddebostan est l'un des points de contact, géographique et moral, où la distance entre Juifs d'Istanbul et «Turcs blancs» est la plus ténue. 
Au moment où s'est instituée la République, espérant à tort «jouir pleinement de leur citoyenneté turque sans avoir à renoncer à leur identité », les Juifs de Turquie se sont trouvés confrontés à un "dilemme identitaire ", lourd d'ambivalences dont Rifat Bali (2010) a bien retracé les linéaments. Le choix du plus grand nombre d'entre eux a été de mimer l'assimilation, de ressembler autant que faire se peut aux "Turcs blancs» tout en maintenant, au sein des foyers et de la communauté, une partie des traditions juives. Cette forme de «marranisation", dans un environnement alors majoritairement turcolaïc à la mode kémaliste, a pendant longtemps pu avoir pour devise "pour vivre heureux vivons cachés » (Kastoryano, 1992a), devise qui reste de mise aujourd'hui encore, malgré certains accommodements (cf. supra et infra). Cet alignement était sans compter sur le nationalisme des «Turcs blancs » (Insel, 2006). Les Juifs d'Istanbul ont trouvé une parade en jouant parallèlement leur rôle classique de minorité intermédiaire, en particulier via leurs activités dans l'économie métropolitaine (Morvan, 2013). Ce rôle s'est aussi porté sur la scène géopolitique internationale ${ }^{10}$. Une telle "intégration politique par l'extérieur » (Kastoryano, 1992c) a été, jusqu'au tournant des années 2000 et l'arrivée au pouvoir de l'AKP à l'échelle nationale, une stratégie plutôt payante, couronnée notamment par l'accord militaire des années 1990 entre la Turquie et Israël, accord qui constituait en retour un gage sécuritaire important pour les Juifs turcs. Néanmoins, comme l'explique Rifat Bali, les dirigeants communautaires doivent, aujourd'hui comme hier, composer avec un "nombre réduit d'options» et une "marge de manœuvre étroite», déplorant que «la marche de la Turquie vers plus de démocratisation et de libéralisation n'ait eu que si peu de conséquences positives à long terme pour la communauté juive du pays » (Bali, 2009). Le processus de turquification a, pour l'essentiel, été un miroir aux alouettes pour les Juifs de Turquie et leurs dilemmes en conséquence paraissent avoir accouché d'autant d'apories.

Malgré leur nom, les "Turcs blancs" ne considèrent nullement les Juifs comme étant des leurs, ni réciproquement ${ }^{11}$. L'un des symptômes de cet état de fait est particulièrement problématique lors de mariages et unions mixtes, de plus en plus nombreux en Turquie, de Juifs avec des non-Juifs. Ces derniers, musulmans ou non-musulmans (en particulier des Arméniens), ont la plupart $\mathrm{du}$ temps en commun leur identification avec un certain nombre de valeurs portées par les «Turcs blancs». Cependant, le partage de ces valeurs ne garantit en rien l'acceptation par les familles «turques blanches» d'alliance matrimoniale avec des Juifs. L'homogamie ne suffit pas ${ }^{12}$. Marcy Brink-Danan (2013)

10. Pour plus de détails, en particulier sur l'auto-instrumentalisation des Juifs d'Istanbul dans l'articulation avec les lobbies juifs américains, voir Bali (2001).

11. Parmi les derniers mots de judéo-espagnols couramment employés figurent des vocables qui servent à l'autodéfinition, comme "nosotros" [nous], ou qui marquent la différence avec les Musulmans, appelés «vedre» [vert]

12. En revanche, sous l'influence des normes de la bourgeoisie turque blanche, cette homogamie a été appliquée à l'intérieur de la communauté juive (Behar, 2006), réduisant drastiquement 
montre bien les grandes difficultés rencontrées, en particulier par les Juives d'Istanbul, dans ce cas de figure. Si loin si proche... La réticence provient du côté turc blanc plus que du côté juif. Malgré leur traditionalisme religieux, ainsi que les réprobations et découragements quasi automatiques contre ces unions, les Juifs d'Istanbul se montrent finalement un peu moins intolérants que les "Turcs blancs " ${ }^{13}$. Une des raisons de ce phénomène paraît tenir à ce que Ayşe Buğra qualifie de « «tache de naissance » [doğum lekesi] propre à la grande bourgeoisie turque, dont une partie des familles a fait fortune grâce aux lois discriminatoires ayant frappé les minorités arménienne, grecque et juive» (Behar, 2011). Cette origine niée semble de nature à aiguiser la violence du «narcissisme de la petite différence» tel qu'il peut s'exprimer dans le contexte turc, comme l'a mis en évidence Benoît Fliche à partir du cas des Alévis : pour se protéger, ceux-ci se voient parfois contraints de recourir à l'art de la dissimulation [takiye] de façon à "apparaître tel que l'on n'est pas et être tel que l'on ne paraît pas », une « manipulation des objets d'identification pour ne pas être identifié » (Fliche, 2014). Cela confère aux Juifs de Turquie forces et faiblesses à la fois: "fort comme le fer, fragile comme une rose ", pour reprendre les mots délicats de Leyla Neyzi (2005). Ainsi, la strate issue du kémalisme et de la turquification, en dépit de son égalisation apparente et de ses ouvertures en clair-obscur, participe d'une rigidification des frontières entre les mondes juifs et non-juifs.

\section{Les nouvelles frontières des communautés imaginées}

Cette dernière strate nous conduit à appréhender de nouvelles urbanités et religiosités en cours, sans la profondeur historique des deux autres strates. En effet, les évolutions décrites dans cette dernière partie remontent à moins d'une génération. Elles sont aussi très liées à l'intense globalisation de la mégapole du Bosphore.

\section{Des communautés imaginées à l'heure des gated communities}

L'Istanbul d'aujourd'hui, avec ses plus de 15 millions d'usagers, a peu à voir avec la métropole de la fin de l'Empire ottoman puis des débuts de la période républicaine. En effet, ses horizons urbains se sont démultipliés: le territoire de l'agglomération croît de 2000 à 500000 hectares et de nouveaux secteurs urbanisés surgissent de toutes parts. Parmi ceux-ci, la forme urbaine qui obtient le plus vif succès auprès des classes moyennes et supérieures est la gated community [en turc: site]. Les Juifs d'Istanbul ne font pas exception

\footnotetext{
l'univers des possibles en matière matrimoniale et compartimentant les couches sociales d'une minorité déjà faible démographiquement.

13. Un juif d'Istanbul, jeune cadre dynamique, m'a ainsi confié avoir été menacé de mort par le père turc blanc de sa petite amie, issu du prestigieux lycée Galatasaray tout comme lui, celui-ci l'accusant de la corrompre, notamment en la droguant.
} 


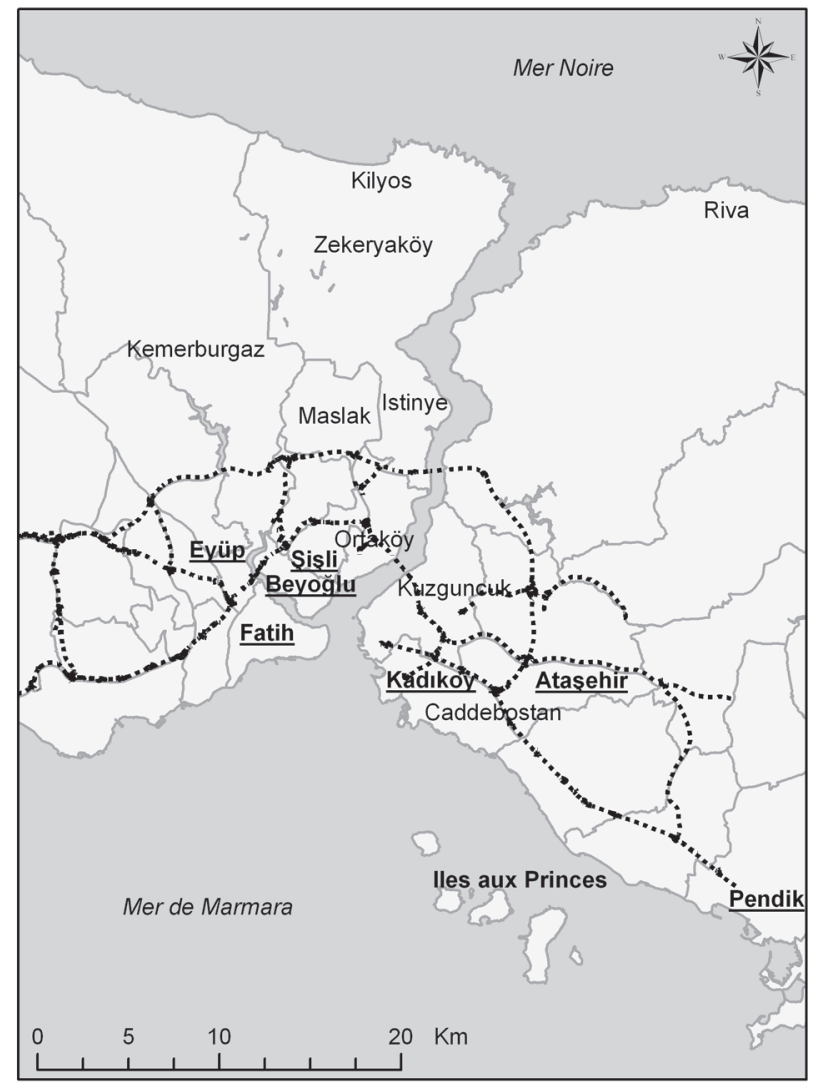

Photo 2. Polarités juives dans la mégapole stambouliote au début du XXI ${ }^{\mathrm{e}}$ siècle, carte de l'auteur

et prisent particulièrement ces complexes résidentiels fermés et sécurisés. Les raisons en sont multiples et ne se distinguent qu'assez peu du reste des consommateurs de ces biens immobiliers, même si certaines site concentrent quelques dizaines de foyers juifs désireux d'avoir des coreligionnaires dans leur voisinage. Le cas d'Alkent à Etiler, analysé par Ségolène Debarre (2004), est paradigmatique: les familles juives y seraient une centaine environ, attirées par l'appartenance du promoteur à la communauté juive et par le bouche-à-oreille, sans que ce réseau soit prépondérant car d'autres effets de club influencent tout autant son peuplement et ses sociabilités, tel celui des anciens élèves du lycée Galatasaray, par exemple. Le renouveau de la synagogue d'Ortaköy doit beaucoup à la proximité de plusieurs zones de gated communities en remontant sur les contreforts du Bosphore. Cela a eu des effets en retour sur les formes communautaires dans et autour de ce lieu de culte, puisqu'il est devenu lui-même une sorte de club. Pendant les travaux de sécurisation suite aux attentats de 2003, la synagogue a été fermée durant une année, les offices 
religieux s'effectuant alors chez les uns et les autres, les rouleaux de la Thora passant d'une gated community à une autre, semaine après semaine, entre Ulus et Etiler (Morvan, 2011b). Depuis lors, la synagogue est très fréquentée le samedi matin avec sa riche collation à la fin de l'office. Elle offre ainsi une sorte de complément à la vie des site, avant qu'une quarantaine de fidèles n'aillent non loin de là, au bord des eaux du Bosphore, deviser des affaires du monde et de l'économie turco-stambouliote au House Café. L'affinitaire et l'hédonisme y priment sur le communautaire stricto sensu ou l'argument sécuritaire, de la même manière que les effets de mode pour les gated communities les plus en vogue.

Hormis pour les plus démunis, la métropolisation des territorialités stambouliotes se caractérise par la multiplication de nouveaux espaces de consommation (shopping malls, plus d'une centaine désormais dans la mégapole) et de résidence (gated communities, déjà 600 en 2009). Ce sont autant d'enclavements choisis qui, à l'instar d'un kaléidoscope, font de la mégapole une juxtaposition polycentrique de parcs à thème en recomposition rapide. Cela signe, entre autres, la fin du rêve "Turc blanc" d'un univers urbain stable et homogénéisé selon leurs critères. Si la fin du kémalisme s'est progressivement accompagnée de la montée de l'islam politique comme modèle souhaitant s'y substituer, l'un et l'autre ont été partiellement contrebalancés par un mouvement parallèle d'assez vaste ampleur : l'essor de la pluralisation des pratiques et des représentations par les effets de la globalisation et des mutations du capitalisme urbain sur les modes de vie. Les Juifs d'Istanbul ont ainsi été happés par ce double mouvement, notamment en matière religieuse. Certains ont accompli un retour à la pratique religieuse orthodoxe, comme chez leurs concitoyens sunnites; tandis que d'autres se sont essayés à diverses pratiques religieuses et parareligieuses, ici aussi à l'image de nombreux stambouliotes de diverses obédiences confessionnelles. Volatiles, ces communautés imaginées instables sont tributaires des dernières tendances de consommation du moment. Elles composent des portfolios à la carte, loin des menus proposés par les deux autres strates étudiées précédemment. Cependant, cette pluralisation n'est, par ailleurs, pas exempte d'une nouvelle quête de sens holistique que les autorités religieuses traditionnelles semblent mal en mesure de proposer de façon adéquate.

\section{La «pop-Kabbalah ${ }^{14}$ », révélatrice de nouvelles aspirations}

La présence à Istanbul d'un groupe de disciples des enseignements de Berg à partir de sa vision (édulcorée) de la kabbale peut, à première vue, sembler assez marginale ${ }^{15}$. En effet, la communauté juive est démographiquement restreinte

14. L'expression est de l'auteur de cet article, forgée en raison de la participation pendant plusieurs années de la chanteuse Madonna aux activités des Kabbalah centre de Berg.

15. L'étude de ce groupe bénéficie de ma participation au programme ANR franco-allemand NeoReligitur, dirigé par Alexandre Toumarkine (Orient Institut Istanbul) et Nathalie Clayer (Cetobac). 
et reste globalement très affiliée aux instances rabbiniques de la minorité. Néanmoins, ces dernières ont au fil du temps (voir supra, les deux précédentes strates) perdu de leur autorité. Les dynamiques de retour à l'orthodoxie religieuse, limitées mais vivaces, ont été guidées par des rabbins étrangers ou turcs vivants à l'étranger. Si l'émissaire du mouvement 'Habad Loubavitch a éprouvé de grandes difficultés à s'implanter dans la vie juive stambouliote, il a fini par exercer une influence notable sur d'assez nombreux fidèles. Cette concurrence religieuse a poussé les rabbins locaux à hausser leur niveau d'enseignement, voire leur degré de respect des lois alimentaires (cacherout), etc. Toutefois, à mesure que cette influence s'est propagée, les Juifs d'Istanbul «ré-orthodoxisés » ont eu tendance à quitter la Turquie, en général pour émigrer vers Israël (alya). De plus, ce processus de ré-enclavement relatif n'est pas du goût de tous et surtout de toutes, les Juives d'Istanbul étant en général plus rétives que les hommes à la pratique orthodoxe du judaïsme. Il ne peut de surcroît que concerner des membres officiellement reconnus de la minorité juive stambouliote et non pas donc, les réprouvés: couples non admis (non-juifs ou homosexuels) ou candidats musulmans au rapprochement d'avec les mondes juifs. Cela laisse de larges pans de ou autour de la communauté juive d'Istanbul sans offre d'enseignements en matière de judaïsme(s). Ces divers éléments de contexte ont constitué les conditions de possibilité de l'émergence d'un groupe de disciples de la Kabbalah de Berg à Istanbul, numériquement modeste mais particulièrement significatif des nouvelles dynamiques de pluralisation ${ }^{16}$.

Le groupe stambouliote de disciples compte environ une centaine de personnes. Il est composé d'un premier cercle d'une trentaine de fidèles auxquels s'adjoignent des visiteurs plus ou moins occasionnels qui gravitent à des degrés divers autour de ce premier cercle. Il comporte une majorité de Juives, parfois accompagnées de leur conjoint. Des non-Juives font également partie des disciples réguliers, dont notamment quelques Sabbataïstes ${ }^{17}$ ou encore des femmes travaillant dans les médias, attirées par l'effet de mode à l'échelle globale. Les leçons se déroulent un soir par semaine, via skype depuis les États-Unis, principalement ${ }^{18}$ dans une proto-gated community des hauteurs $\mathrm{du}$ Bosphore, anciennement coopérative de journalistes [gazeteciler sitesi]. Elles ont lieu à Safir, un centre proposant une large gamme d'activités allant de la thérapie gestaltiste et des cures par la danse ou les massages, jusqu'au taïchi et au yoga, en passant par la "détox». L'environnement immédiat de Safir est, quant à lui, formé d'entreprises technologiques ou à vocation créatives. Istanbul y prend des faux airs de Beverly Hill. Los Angeles est la

\footnotetext{
16. Étudiées par l'ANR NeoReligitur, ces nouvelles dynamiques concernent l'ensemble des populations de Turquie, musulmans sunnites compris. Concomitantes de l'homogénéisation promue par l'islam politique au pouvoir actuellement, elles témoignent de la complexification à l'œuvre, notamment en matière religieuse, au sein de la société turque.

17. Musulmans d'origines juives ayant suivi le nouveau messie Sabbataï Zevi.

18. Un plus petit groupe se réunit à Göktürk, un autre secteur de l'agglomération d'Istanbul constitué uniquement de gated communities (Pérouse, 2004).
} 
mégapole où s'origine le mouvement initié par Berg, aidé par sa femme qui lui a succédé après son décès (2013). Affaire de femmes donc... qu'à Istanbul, la vie communautaire juive n'a sans doute pas assez intégrées en matière d'enseignements herméneutiques et holistiques du judaïsme. La frontière est ici celle du genre et la Kabbalah de Berg y fait office de passeur pour elles et pour certains non-Juifs désireux d'être en lien avec un judaïsme non rabbinique ${ }^{19}$. L'anthropologue Véronique Altglas (2014) montre bien comment les centres Kabbalah représentent des formes de «synagogue inclusive». Cependant, le paradoxe est que cette inclusion s'effectue à Istanbul en contexte fermé, celui des site, dont le maître mot est en turc özel, " exclusif ». Les porosités que la "pop-Kabbalah» permet se trouvent limitées par cette exclusivité. Le cas de ce groupe est ainsi révélateur de nouveaux types, urbains et moraux, de partage des frontières des mondes juifs. Il témoigne aussi de la difficulté des autorités religieuses traditionnelles, avec leurs conceptions héritées et parfois arrêtées des frontières, à pourvoir une récollection fluide ${ }^{20}$ aux subjectivités divisées des métropoles contemporaines que les urbanités pluralisées suscitent.

\section{$* * *$}

Le parcours de la minorité juive d'Istanbul, de par ses frontières propres et celles rencontrées au fil des strates successives, représente un baromètre de l'évolution de la société turco-stambouliote. La trajectoire urbaine et commerciale de la firme Vakko en est un indicateur. En effet, Vitali Hakko tenait une modeste boutique de chapeaux à Péra, établie en 1934. Son premier magasin de prêt-à-porter ouvre ses portes en 1962 non loin de là, tandis qu'une usine commence à produire à partir de 1968 à Merter, dans ce qui était alors une périphérie industrielle à proximité de l'aéroport; quarante ans plus tard, c'est à Esenyurt, sur les franges occidentales de l'immense mégapole stambouliote, que Vakko inaugure son nouveau centre de production. Fleuron du textile de luxe accompagnant les évolutions ambivalentes de la société turque, la marque propose aujourd'hui aussi bien des voiles que de très courtes minijupes ${ }^{21}$. La minorité juive est contrainte de s'adapter. Si, dans le meilleur des cas, il peut s'agir d'une "tension créatrice » faisant de la minorité une sorte de "poisson pilote» (Morvan, 2013), elle doit en toutes circonstances naviguer dans le «jeu trouble» des représentations complexes et souvent péjoratives (Mallet, 2011) des contextes successifs ottomano-turcs. Cela a conduit de larges proportions

19. Il faut noter ici l'absence à Istanbul, pour des raisons historiques, des courants libéraux et conservatives.

20. Là encore, comme face à la concurrence du mouvement 'Habad, le judaïsme stambouliote tente de rivaliser. Ainsi le rabbin de la synagogue d'Ortaköy, espace cultuel auquel le groupe de disciple de Berg est lié puisque une dizaine de membres de son noyau dur s'y rend chaque samedi matin, propose-t-il désormais des cours mixtes précédés d'un cocktail sur la réincarnation, pensant de la sorte répondre à leur demande de spiritualité.

21. Vakko possède également trois magasins hors de Turquie : deux en Allemagne et un à Abu Dhabi, reflétant les connections économiques globalisées de la métropole turque. 
de Juifs d'Istanbul à quitter la Turquie, ou à s'inscrire dans des mobilités transnationales. Sur cet état de fait s'articulent également les frontières sociales internes à la minorité juive, mouvantes au gré de ces strates et départs. Cela invite à poursuivre la réflexion dans la perspective adoptée par Georg Simmel (2010), pour lequel «la frontière n'est pas un fait spatial avec des conséquences sociologiques, mais un fait sociologique qui prend une forme spatiale ». L'humain est un «être de frontière qui n'a pas de frontière "(Simmel, 1993).

Yoann MORVAN

Institut d'ethnologie méditerranéenne, européenne et comparative (IDEMEC)

Aix Marseille Université

yoannmorvan@yahoo.com

\section{Bibliographie}

Aквulut Rifat, 2010, « Kadiköy. L'autre ville de l'autre côté », Urbanisme, n 374, p. 43-45. Altglas Véronique, 2014, From Yoga to Kabbalah. Religious exoticism and the logics of bricolage, Oxford University Press.

BALI Rifat, 2001, Les relations entre Turcs et Juifs dans la Turquie moderne, Istanbul, Isis. —, 2009, «Entre nationalisme et islamisme: la lente disparition de la communauté juive de Turquie », Trigano S. (dir.), La fin du judaïsme en terres d'Islam, Paris, Denoël.

—, 2010, «Le dilemme identitaire des Juifs dans les premières années de la République turque », Benbassa E. (dir.), Itinéraires séfarades. Complexité et diversité, Paris, Presses de l'Université Paris-Sorbonne, p. 127-140.

Barkey Karen, Gravilis George, 2016, “The Ottoman Millet System: Non-Territorial Autonomy and its Contemporary Legacy”, Ethnopolitics, 15, 1.

BeHAR David, 2006, "L'inscription de l'héritier dans la lignée. Filiation, affiliation et réussite sociale en grande bourgeoisie turque ", European Journal of Turkish Studies, $\mathrm{n}^{\circ} 4$.

—, 2008, «La notion de bourgeoisie dans l'historiographie de la Turquie contemporaine ", Revue des mondes musulmans et de la Méditerranée, no 123, p. 205-226.

—, 2011, «La troisième génération de la grande bourgeoisie turque », Cahiers de la Méditerranée, no 82, p. 231-250.

Braude Benjamin, Lewis Bernard, 1982, Christian and Jews in the Ottoman Empire: the Functioning of a Plural Society, New York, Londres, Holmes \& Meier.

BRINK-DANAN Marcy, 2005, Reference points: text, context and change of definitions of turkish-jewish identity, thèse de doctorat [Ph.D.], Palo Alto, Californie, Stanford University.

—, 2010a, «Exposer la tolérance. La différence et le doute dans un musée en Turquie », Benbassa E. (dir.), Itinéraires séfarades. Complexité et diversité, Paris, Presses de l'Université de Paris-Sorbonne, p. 141-155.

—, 2010b, "Counting as European: Jews and the Politics of Presence in Istanbul", Göktürk D., Soysal L., Türeli I. (eds.), Orienting Istanbul: Cultural Capital of Europe? London, Routledge. 
—, 2013, “Love, Luck and Long Distances. Choice and Chance in Turkish Women's Emigration Stories”, Ethnologie française, 4, vol. 43, p. 615-622.

CoHen Julia, 2014, Becoming Ottomans: Sephardi Jews and Imperial Citizenship in the Modern Era, Oxford University Press.

Debarre Ségolène, 2004, Alkent, une gated community alaturka? Conditions d'application d'un modèle américain en Turquie, mémoire de maîtrise en géographie, Université Lyon 2.

Fliche Benoit, 2014, “Narcissisme de la petite différence” et intolérances religieuses. Le cas des alévis et des sunnites en Anatolie centrale", Identité et politique. De la différenciation culturelle au conflit, Paris, Presses de Science Po.

GüLERYÜz Naim, 2008, The synagogues of Istanbul, Istanbul, Gözlem.

—, 2009, The turkish Jews, 700 years of togetherness, Istanbul, Gözlem.

Insel Ahmet, 2006, "Le nationalisme des “Turcs blancs” ", La Vie des idées, no 14.

KARMI Ilan, 1992, Jewish sites of Istanbul, Istanbul, Isis Press.

Kastoryano Riva, 1992a, «Du Millet à la communauté: les Juifs de Turquie », Pardès, vol. 15, 1992, p. 137-158.

—, 1992b, «Trajectoires dans la ville: les Juifs à Istanbul», Critique, vol. 543-544, p. 657-670.

—, 1992c, «L'intégration politique par l'extérieur. La communauté juive de Turquie ", Revue française de science politique, $42^{\mathrm{e}}$ année, $\mathrm{n}^{\mathrm{0}} 5$, p. 786-801.

KöKsal Aykut, 2010, «Petite histoire de l'architecture stambouliote ", Urbanisme, $\mathrm{n}^{\circ} 374$, p. 56-59.

Levi Mario, 2011, Istanbul était un conte, Paris, Sabine Wespieser.

Levy Avigdor, 2002, Jews, Turks, Ottomans. A Shared history, Fifteenth Through the Twentieth Century, Syracuse University Press, New York.

Levy-Aksu Noémi, 2013, Ordre et désordres dans l'Istanbul ottomane (1879-1909), Paris, Karthala.

MALLET Laurent, 2011, «Jeu trouble: les complexes représentations du Juif en Turquie », Les Cahiers du judaïsme, $\mathrm{n}^{\circ}$ 32, p. 62-73.

Mills Amy, 2010, Streets of memory. Landscape, tolerance, and national identity in Istanbul, Athens, Georgia, The University of Georgia Press.

Montabone Benoit, Morvan Yoann, 2011, «Istanbul: la carte du troisième pont sur le Bosphore », EspacesTemps.net, Objets, 18.04.2011.

Morvan Yoann, 2011a, «La vie juive à Istanbul: entre post-kémalisme et néo-ottomanisme ", Regard sur l'Est.

—, 2011b, «Juifs d'Istanbul, territorialités d'une communauté entre recompositions et dislocations ", ÉchoGéo, n 16.

—, 2013, «Géopolitique et métropolisation, le rôle de "minorité intermédiaire » des juifs d'Istanbul », Espaces et sociétés, n' 154.

Neyzi Leyla, 2005, "Strong as Steel, Fragile as a Rose: A Turkish Jewish Witness to the Twentieth Century”, Jewish Social Studies, vol. XII, no 1, p. 167-189.

Pérouse Jean-François, 2004, «Les “cités sécurisées” des territoires périphériques de l'arrondissement d'Eyüp (Istanbul) ou les mirages de la distinction », L'information géographique, $\mathrm{n}^{\circ}$ 2, p. 139-154. 
—, 2005, «Les non musulmans à Istanbul aujourd'hui: une présence en creux ? Le cas de l'arrondissement de Fatih ", Revue des mondes musulmans et de la Méditerranée, $\mathrm{n}^{\text {os }}$ 107-110, «Identités confessionnelles et espace urbain en terres d'Islam », p. 261-295.

—, 2010, «Istanbul depuis 1923: la difficile entrée dans le xx siècle ? ", Monceau N. (dir.), Istanbul. Histoire, promenades, anthologie \& dictionnaire, Paris, Robert Laffont, p. 231-290.

SENi Nora, 2006, "Istanbul Mahalle and Venitian Ghetto. Is the comparison relevant?”, Eyüp Özveren, Oktay Özel, Süha Ünsal, Kudret Emiroglu (eds.), The Mediterranean World; The idea, The Past and the Present, Istanbul, Iletisim, p. 161-172.

Simmel Georg, 1993, La tragédie de la culture, Paris, Payot.

—, 2010 [1908], Sociologie, Paris, Presses universitaires de France, Quadrige.

SHaw Stanford, 1991, Jews of the Ottoman Empire and the Turkish Republic, Londres, Macmillan.

Trivilatto Francesca, 2009, The Familiarity of Strangers, Yale University Press.

VArol Marie-Christine, 1989, Balat, faubourg juif d'Istanbul, Istanbul, Isis Press.

\section{Juifs d'Istanbul : du parcours minoritaire aux porosités exclusives}

Depuis la fin de l'Empire ottoman, la minorité (millet) juive d'Istanbul a principalement résidé dans des quartiers (mahalle) spécifiques, sans que ces derniers ne fassent figure de ghetto. Avec le passage à la République kémaliste, le nouveau contexte statutaire et politique a imposé aux Juifs turcs d'importantes mutations identitaires. Ceux-ci ont progressivement délaissé leurs quartiers d'origine au profit de nouvelles polarités urbaines, participant à la modernisation d'Istanbul à l'instar des nouvelles élites kémalistes. La perte progressive de poids de cette élite au sein de la Turquie post-kémaliste a entrainé les Juifs d'Istanbul à se repositionner dans un environnement plus complexe que révèlent leurs nouvelles stratégies résidentielles: mixte d'homogénéisation et de différenciation sur fond d'enclavement. Les groupes de Juifs d'Istanbul se caractérisent de plus en plus par leurs porosités exclusives, analysées ici au prisme de l'étude des disciples, surtout féminines et incluant Juifs et non Juifs, de la Kabbalah de Berg.

Mots-clés : Istanbul, (post-)ottoman, judaïsmes, nouveaux mouvements religieux (NMR), espaces urbains.

\section{Istanbul's Jews: from a minority trajectory to exclusive porosities}

The paper retraces the urban trajectory and the evolution of the identity of Jews of Istanbul from the end of Ottoman Empire until today. The Jewish minority (millet) lived for centuries in specific neighbourhoods (mahalle), nevertheless, they were not ghettos. With the arrival of the Kemalist Republic, new statuses where imposed on minorities, forcing the Jewish identity to mutate. The Jews left their traditional neighbourhoods, moving to new urban polarities, participating to the modernisation of Istanbul, following the example of the 
Kemalist elite. The decline of these elites in post Kemalist Turkey had an impact on the Jews of Istanbul. This new and more complex context is revealed by their residential strategies: dialectics between homogenization/differentiation in a metropolis where enclave/gated community is the standard for these urban upper classes. The different groups of Jews of Istanbul are increasingly characterised, simultaneously by forms of porosity, and exclusion. This paradox is analysed through the study case of local disciples of the Berg's Kabbala, a predominantly feminine group, inclusive to Jews and non-Jews.

Key words: Istanbul, (post-)Ottoman, Judaism, new religious movements (NMR), urban spaces.

\section{Judíos de Estambul: del recorrido minoritario a las porosidades exclusivas}

Desde el fin del Imperio Otomano, la minoría (millet) judía de Estambul residió principalmente en barrios específicos (mahalle), sin que estos barrios se constituyeran en la figura del ghetto. Con el pasaje a la república kemalista, el nuevo contexto estatutario y político impuso a los Judios turcos importantes mutaciones identitarias. Éstos dejaron progresivamente sus barrios de origen en provecho de nuevas polaridades urbanas, participando de la modernización de Estambul dirigida por las nuevas élites. La pérdida progresiva de peso de esta élite en el seno de la Turquía post-kemalista llevó a los judios de Estambul a reposicionarse en un ambiente más complejo evidenciado por sus nuevas estrategias residenciales, mezcla de homogeneización/diferenciación sobre un fondo de enclave. El grupo de Judios de Estambul se caracterizan cada vez más por sus porosidades exclusivas, analizadas aqui desde el estudio de los discipulos, sobre todo mujeres, de la Kabbalah de Berg, e incluyendo judios y no judios.

Palabras clave: Estambul, (post)otomano, judaísmos, nuevos movimientos religiosos (NMR), espacios urbanos. 